\title{
A Rare Case of Eosinophilic Gastroenteritis in a Neonate with Fever
}

\author{
Minkyeong Lee, $\mathrm{MD}^{1}$, So Yoon Choi, $\mathrm{MD}^{1}$, Mi Lim Chung, $\mathrm{MD}^{1}$, Jonghyun Lee, $\mathrm{MD}^{2}$, and Myongsoon Sung, $\mathrm{MD}^{2}$ \\ ${ }^{1}$ Department of Pediatrics, Inje University Haeundae Paik Hospital, Busan, Korea \\ ${ }^{2}$ Department of Pediatrics, Soonchunhyang University Gumi Hospital, Gumi, Korea
}

\section{ABSTRACT}

Eosinophilic gastroenteritis is a member of eosinophilic gastrointestinal (GI) diseases and is characterized by the accumulation of eosinophils within the GI tract. Common symptoms of eosinophilic gastroenteritis are vomiting, abdominal pain, dysphagia, and weight loss, but rare fever in neonate. This report describes a 10-day-old boy who developed fever, bloody mucoid stool, and bilious vomiting resulting from eosinophilic gastroenteritis, mimicking sepsis. Sigmoidoscopy and pathologic findings revealed mucosal edema, small hemorrhagic spot, and segmental erythemoid edema in the colon and increased number of eosinophils in the lamina propria with intraepithelial eosinophils. After breast milk feeding with restriction of milk from the mother for 6 months, specific immunoglobulin to milk was class 0 (class 3 , previous). The boy restarted formula feeding, and he had no fever and GI symptoms.

Key Words: Eosinophilic enteropathy; Fever; Infant, newborn

\section{INTRODUCTION}

Gastrointestinal (GI) food allergy is divided into four categories: food protein-induced enterocolitis syndrome, food protein-induced proctocolitis, food protein-induced enteropathy, and eosinophilic GI disorders ${ }^{1)}$. Eosinophilic gastroenteritis is a rare condition characterized by the presence of GI symptoms and eosinophilic infiltration in the GI tract

${ }^{2-5)}$. Depending on the type of injury, it can be further divided into three categories: eosinophilic gastritis, eosinophilic enteritis, and eosinophilic colitis $^{3-5)}$. The epidemiology of eosinophilic gastroenteritis differs among studies, and this condition presents irrespective of age and race, with a higher incidence in men $^{3-5}$. The clinical symptoms may vary depending on the location, depth, and extent of bowel wall involvement and may have a chronic course $^{4,5)}$. Thus, the clinical management of eosinophilic gastroenteritis is difficult, owing to its enigmatic nature and unknown etiology. Moreover, most reports have described eosinophilic gastroenteritis in adults, owing to its rarity in the pediatric population, especially in neonates. Here, we identified a male neonate with eosinophilic gastroenteritis along with fever and bloody stools; we analyzed his clinical course, response to treatment, and resolu-
Received: 26 April 2019

Revised: 10 July 2019

Accepted: 10 July 2019

Correspondence to: Myongsoon Sung, $\mathrm{MD}$

Department of Pediatrics, Soonchunhyang University Gumi Hospital, 179 lgongdan-ro, Gumi 39371, Korea

Tel: +82-54-468-9114

Fax: +82-54- 468-9114

E-mail: myong47@hanmail.net

Copyright(c)

By Korean Society of Neonatology.

All right reserved.

This is an Open-Access article distributed under the terms of the Creative Commons Attribution Non-Commercial License (http://creativecommons.org/licenses/ by-nc/4.0), which permits unrestricted non-commercial use, distribution, and reproduction in any medium, provided the original work is properly cited. 
tion of the gastroenteritis by restriction of the allergen for 6 months.

\section{CASE REPORT}

A 10-day-old male infant visited our tertiary center for evaluation of progressive fever and bloody mucoid stools in January 2018. He was born by vaginal delivery at a local hospital at 38 weeks of gestation (weighing 3,300 g), without any perinatal problems. Both parents had a history of allergic rhinitis. The bloody stool of the patient was noticed about 3 days after birth and feeding of breastmilk; however, the frequency of the stool was once a day. The patient did not have fever that time, but his oral intake was poor. However, the bloody mucoid stools per sisted, and a local pediatrician recommended replacing breastmilk with formula. However, at 5 days of age, the patient had passed bloody mucoid stools over 10 times. Subsequently, he was admitted to a local hospital and started on antibiotic medication (third-generation cephalosporin) under a suspicion of enteritis. Despite antibiotic treatment for 3 days at the local hospital along with formula feeding four times a day, fever, ag gravation of bilious vomiting, abdominal distension, weight loss, and persistent bloody stools were observed. Subsequently, the patient was referred to our center. During physical examination, general weakness and an abnormal abdomen with decreased bowel sound, resulting in severe bowel distension, were observed.

Owing to poor oral intake, the weight of the patient had decreased to $2,830 \mathrm{~g}$, representing a $14.2 \%$ weight loss from the birth weight (3,300 g). The white blood cell (WBC) count, platelet count, C-reactive protein (CRP), fibrin degradation product, Ddimer, and anti-thrombin III level were $21,360 / \mu \mathrm{L}(30.0 \%$, neutrophils), 415,000/ $\mu \mathrm{L}, 14.13 \mathrm{mg} / \mathrm{dL}, 13.3 \mu \mathrm{g} / \mathrm{mL}, 4.29 \mu \mathrm{g} / \mathrm{mL}$, and $57.7 \%$, respectively, suggestive of systemic infection and disseminated intravascular coagulation (DIC). However, blood culture showed no bacteria. Stool analysis revealed the presence of red blood cells (>100 ng/mL); however, no viruses, bacteria, or parasites were observed in the polymerase chain reaction test and culture. Sonographic examination of the abdomen revealed diffuse bowel wall thickening of the small bowel and colon, and nonspecific enterocolitis, including necrotizing enterocolitis, was ruled out (Figure 1A).

Total immunoglobulin E (IgE) and eosinophil count levels were elevated at $32.6 \mathrm{IU} / \mathrm{mL}$ and $4,701 / \mu \mathrm{L}$, respectively, and peripheral blood analysis revealed the presence of $7 \%$ eosinophils. Specific IgE (sIgE) to milk was class 3 (5.11 kUA/L), and skin prick test to milk showed positive results. Sigmoidoscopy established a diagnosis of eosinophil gastroenteritis, revealing mucosal edema, small hemorrhagic spots, and segmental erythematous edema in the colon (Figure 1B, C). Biopsy showed several eosinophils in the lamina propria (up to 90/high power field [HPF]), with intraepithelial eosinophils (up to 10/HPF) (Figure 2).

Intravenous administration of vancomycin, meropenem, and metronidazole, without any oral medication, for 5 days resulted in resolution of fever, bloody mucoid stools, and bilious vomiting. Additional treatment with intravenous immunoglobulin and anti-thrombin III markedly improved the fever, mucoid bloody stools, and general condition of the patient. After the 5-day treatment at our hospital, the WBC count and CRP level of the patient were $28,840 / \mu \mathrm{L}(30.0 \%$ neutrophils, $16.3 \%$ eosinophils $)$ and $1.89 \mathrm{mg} / \mathrm{dL}$, respectively. Sonographic examination of the

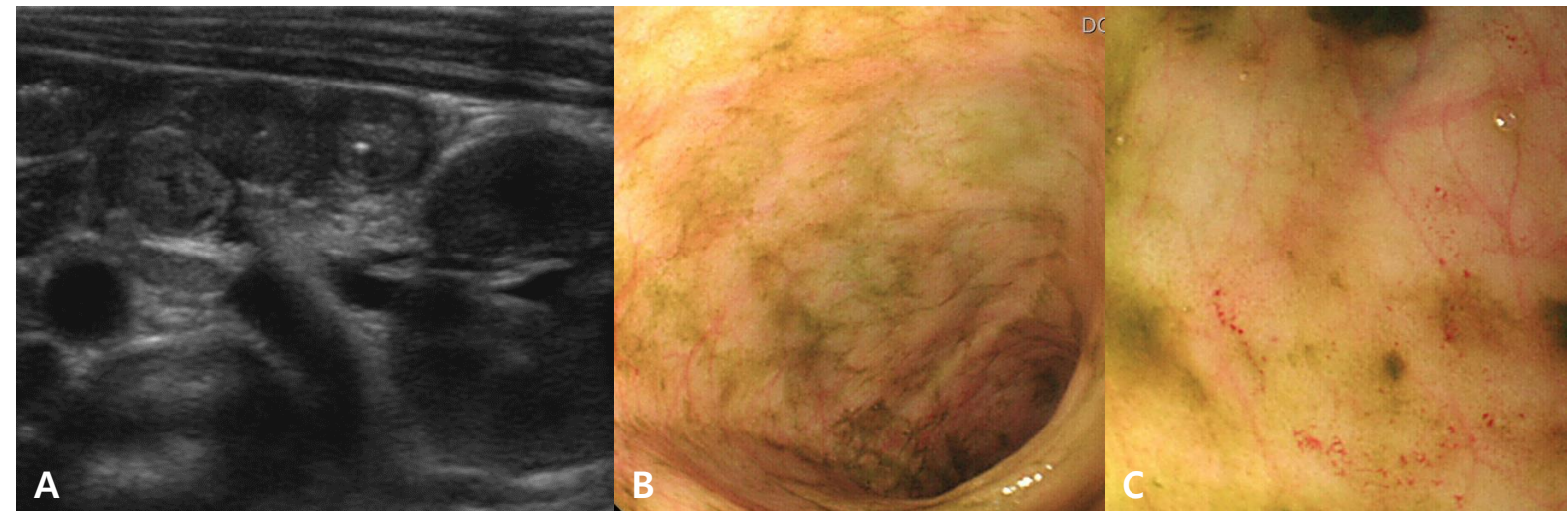

Figure 1. Abdominal ultrasonography in a male neonate with diffuse bowel wall thickening of the small bowel and colon (A), and sigmoidoscopy findings showing mucosal edema, small hemorrhagic spot, and segmental erythematous edema in the rectum and sigmoid colon $(B, C)$. 


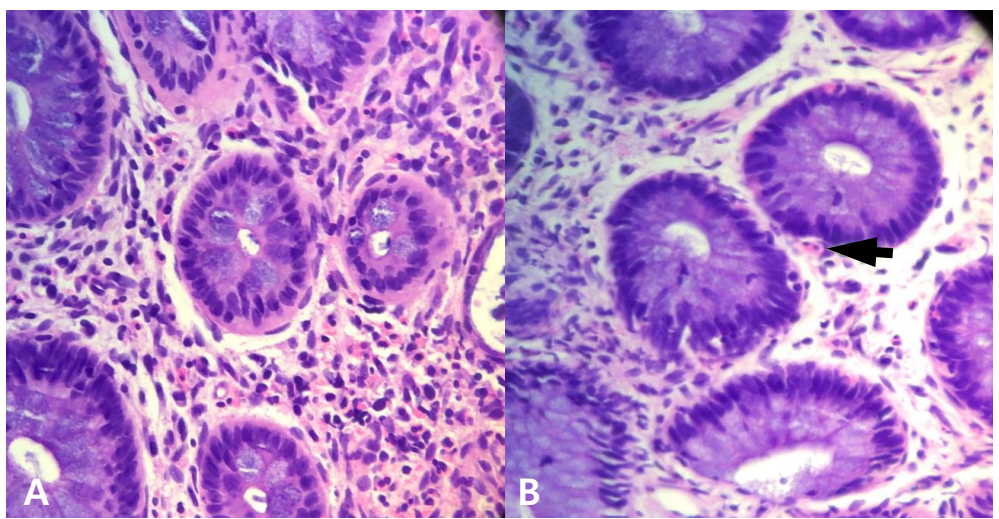

Figure 2. (A, B) Microscopic findings in a male neonate with increased number of eosinophils in the lamina propria (up to 90/high power field [HPF]), with intraepithelial eosinophils (up to 10/HPF) and minimal acute and chronic inflammation in the colon $(\mathrm{H} \& \mathrm{E}, \times 200)$ (arrow: eosinophil).

abdomen revealed absence of diffuse bowel wall thickening of the small bowel and colon, and an improved status compared to that observed in the previous examination. The patient was fed a hypoallergenic formula (HA ${ }^{\circledR}$, Maeil Dairies Co. Ltd., Seoul, Korea); this time, no fever, mucoid bloody stools, or vomiting was observed. On admission day 9, the patient was fed breastmilk and the hypoallergenic formula, while restricting the consumption of cow milk by the mother.

Fortunately, the patient no longer presented with fever or adverse GI symptoms. After discharge from our hospital, his diet for the next 6 months consisted of breastmilk, with the mother advised to restrict the consumption of cow milk.

After 6 months, the patient revisited our clinic, and physical examination showed that his weight and height were 8,400 $\mathrm{g}$ and $70.4 \mathrm{~cm}$, respectively, which were within normal ranges. The total serum IgE and eosinophil count had decreased to $2.2 \mathrm{IU} /$ $\mathrm{mL}$ and $801 / \mu \mathrm{L}$, respectively. A sIgE to milk was class 0 , and peripheral blood analysis revealed the presence of $10 \%$ eosinophils, which was an improvement from the results observed 6 months ago. Formula feeding was resumed, but the patient did not develop GI symptoms or fever.

Based on the findings of sigmoidoscopy and pathological examination, the 10-day-old neonate who presented with high fever, bilious vomiting, mucoid bloody stool, and abdominal distension was diagnosed with eosinophil gastroenteritis, and sepsis caused by an infection was ruled out.

\section{DISCUSSION}

We report a case of eosinophilic gastroenteritis in a male neonate with fever and DIC, mimicking necrotizing enterocolitis or sepsis, who was completely cured by intravenous antibiotic administration and diet restriction.

Eosinophilic gastroenteritis is characterized by the accumulation of eosinophils within the GI tract ${ }^{1,2)}$. According to a recent study, although the estimated incidence of eosinophilic gastroen teritis remains relatively low in the United States, it has been in creasing in recent years ${ }^{1)}$. However, this estimation of eosinophilic gastroenteritis may be inaccurate, as many cases are not accu rately diagnosed or reported in patients. Based on the organs affected and the intensity of eosinophilic inflammation, various GI symptoms may occur ${ }^{1-3)}$. The common GI symptoms include vomiting, abdominal pain, diarrhea, and weight loss ${ }^{1,2)}$; however, fever and DIC, as observed in the neonate in this study, are rarely observed. These common symptoms are similar to those of en teritis by infection; this may delay the exact diagnosis in some patients. Although the etiology and pathophysiology underlying eosinophil gastroenteritis are currently unknown, eosinophils may play a crucial role in the mechanism of tissue injury. Therefore, atopy and food allergy appear to play a role ${ }^{5,6)}$, as observed in this case, in milk allergy.

The diagnosis of eosinophilic gastroenteritis is based on the presence of GI symptoms, eosinophilic infiltration in the GI tract, typical radiological findings, peripheral eosinophilia, and the absence of parasitic or extra-intestinal disease ${ }^{7)}$. While patients with eosinophilic gastroenteritis may have elevated serum IgE levels or hypereosinophilia in blood, these symptoms alone may 
be insufficient for the diagnosis of eosinophilic gastroenteritis ${ }^{5,6}$. Thus, biopsies from various sites and eosinophil count are more relevant for the diagnosis of this disease. Similar to our observations presented here, there is a reported case of a patient with eosinophilic gastroenteritis and colonoscopy findings showing mucosal edema and erosions, small hemorrhagic spot, and erythematous edema and hyperemia in the colon ${ }^{8)}$.

Moreover, allergic proctocolitis diagnosed in infancy is considered an overall transient and benign condition. The symptoms of proctocolitis usually develop in the first 2 to 8 weeks of life, but they can occur in the first few days in rare cases ${ }^{9}$. Allergic proctocolitis is associated with a personal or family history of allergy $^{10)}$. The main feature in allergic proctocolitis is blood with or without mucus in the stools of an otherwise normally growing and developing infant ${ }^{10)}$. Although acute symptoms of allergic proctocolitis have been noted after ingestion of the offending food ( $<12$ hours), the symptoms are more likely to develop gradually over time ${ }^{11)}$. In clinical practice, allergic proctocolitis is diagnosed when resolution of symptoms with dietary avoidance and re-occurrence of symptoms with re-introduction are observ$\mathrm{ed}^{12}$. Skin prick testing and sensitization tests are typically negative to the suspected foods ${ }^{9-12)}$.

It is well established that the treatment of eosinophilic gastroenteritis is empirical. Diet restriction, administration of systemic or topical corticosteroids, and immune-modulatory therapies have been used to treat eosinophilic gastroenteritis ${ }^{13,14)}$. Therefore, there is no standard therapy for this condition. If food allergens are suspected or confirmed by blood test, skin prick test, food challenge test, or clinical symptoms, food restriction should be considered. Although food restriction suggested by skin tests has shown uncertain effects, amino acid-based elemental diets resolved both symptoms and tissue eosinophilia in patients with eosinophil gastroenteritis ${ }^{15}$. However, the main drawbacks of dietary restriction may involve recurrence of symptoms with reintroduction of non-elemental foods, nutritional imbalance, and mental burden on patients and their families. Systemic or topical corticosteroids have shown favorable results in patients with eosinophilic gastroenteritis in whom dietary treatment is not feasible or effective ${ }^{16,17}$. Long-term use of systemic corticosteroids is limited because of the risk of systemic side effects; hence, many patients with eosinophilic gastroenteritis do not achieve complete symptom remission. This points to food restriction and corticosteroid treatment as efficient methods for improvement of clinical symptoms in most cases; however, persistent medical treatment and follow-up are required. In the present case, we found that the condition of the neonate rapidly improved with dietary therapy alone, and corticosteroid therapy was not required. This finding is similar to the result of another study in which seven patients showed clinical improvement with dietary therapy alone, and one patient improved after antihistamine therapy and food restriction, without the requirement of corticosteroid therapy ${ }^{18)}$. In summary, this case shows that a neonate with eosinophilic gastroenteritis presenting with septic condition and DIC, along with high fever, bilious vomiting, and bloody stools, mimicking sepsis due to infection, can be successfully cured with food restriction for 6 months.

\section{ARTICLE INFORMATION}

\section{Ethical statement}

The study protocol was approved by the Institutional Review Board of the Haeundae Paik hospital (IRB No. 2019-10-017). Informed consent was obtained from the patient's parents.

\section{Conflicts of interest}

No potential conflict of interest relevant to this article was reported.

\section{Author contributions}

Conceptualization: M.S.

Acquisition, analysis, or interpretation of data: M.L., M.S.

Drafting the work or revising: M.L.

Final approval of the manuscript: S.Y.C., M.L.C., J.L.

\section{ORCID}

Minkyeong Lee https://orcid.org/0000-0002-5767-6808

Myongsoon Sung http://orcid.org/0000-0002-6329-286X

\section{Acknowledgments}

None

\section{REFERENCES}

1. Spergel JM, BookWM, Mays E, Song L, Shah SS, Talley NJ, et al. Variation in prevalence, diagnostic criteria, and initial management options for eosinophilic gastrointestinal diseases in the 
United States. J Pediatr Gastroenterol Nutr 2011;52:300-6.

2. Guajardo JR, Plotnick LM, Fende JM, Collins MH, Putnam PE, Rothenberg ME. Eosinophil-associated gastrointestinal disor ders: a world-wide-web based registry. J Pediatr 2002;141:57681.

3. Whitaker IS, Gulati A, McDaid JO, Bugajska-Carr U, Arends MJ. Eosinophilic gastroenteritis presenting as obstructive jaundice. Eur J Gastroenterol Hepatol 2004;16:407-9.

4. Treiber GG, Weidner S. Eosinophilic gastroenteritis. Clin Gastroenterol Hepatol 2007;5:e16.

5. Rothenberg ME. Eosinophilic gastrointestinal disorders (EGID). J Allergy Clin Immunol 2004;113:11-28; quiz 29.

6. Chehade M, Aceves SS. Food allergy and eosinophilic esophagitis. Curr Opin Allergy Clin Immunol 2010;10:231-7.

7. Lucendo AJ, Arias A. Eosinophilic gastroenteritis: an update. Expert Rev Gastroenterol Hepatol 2012;6:591-601.

8. Abassa KK, Lin XY, Xuan JY, Zhou HX, Guo YW. Diagnosis of eosinophilic gastroenteritis is easily missed. World J Gastroenterol 2017;23:3556-64.

9. Sopo SM, Filoni S, Giorgio V, Monaco S, Onesimo R. Food protein-induced enterocolitis syndrome (FPIES) to corn: a case report. J Investig Allergol Clin Immunol 2012;22:391-2.

10. Nomura I, Morita H, Hosokawa S, Hoshina H, Fukuie T, Watanabe M, et al. Four distinct subtypes of non-IgE-mediated gastrointestinal food allergies in neonates and infants, distinguished by their initial symptoms. J Allergy Clin Immunol 2011;127:685-8.

11. Fogg MI, Brown-Whitehorn TA, Pawlowski NA, Spergel JM.
Atopy patch test for the diagnosis of food protein-induced enterocolitis syndrome. Pediatr Allergy Immunol 2006;17:351-5.

12. Eigenmann PA, Belli DC, Ludi F, Kahn JM, Polla BS. In vitro lymphocyte proliferation with milk and a casein-whey protein hydrolyzed formula in children with cow's milk allergy. J Allergy Clin Immunol 1995;96:549-57.

13. Bolukbas FF, Bolukbas C, Uzunkoy A, Baba F, Horoz M, Ozturk E. A dramatic response to ketotifen in a case of eosinophilic gastroenteritis mimicking abdominal emergency. Dig Dis Sci 2004;49:1782-5.

14. Bischoff SC. Food allergy and eosinophilic gastroenteritis and colitis. Curr Opin Allergy Clin Immunol 2010;10:238-45.

15. Chehade M, Magid MS, Mofidi S, Nowak-Wegrzyn A, Sampson HA, Sicherer SH. Allergic eosinophilic gastroenteritis with protein-losing enteropathy: intestinal pathology, clinical course, and long-term follow-up. J Pediatr Gastroenterol Nutr 2006;42: 516-21.

16. Tan AC, Kruimel JW, Naber TH. Eosinophilic gastroenteritis treated with non-enteric-coated budesonide tablets. Eur J Gastroenterol Hepatol 2001;13:425-7.

17. Siewert E, Lammert F, Koppitz P, Schmidt T, Matern S. Eosinophilic gastroenteritis with severe protein-losing enteropathy: successful treatment with budesonide. Dig Liver Dis 2006; 38:55-9.

18. Choi BS, Hong SJ, Park SH, Kim HM, Choe BH. Differences in features and course of mucosal type eosinophilic gastroenteritis between Korean infants and children. J Korean Med Sci 2015; 30:1129-35. 